\title{
Conservación de centros históricos. Métodos para estudios previos
}

\author{
Lourdes Gómez Consuegra ${ }^{1}$
}

\begin{abstract}
Resumen: El diseño de algoritmos y la elaboración de métodos automatizados para obtención de la información básica necesaria para el planeamiento urbano de un centro histórico fue en la década de los 80's una gran novedad científica cuyo aporte principal estuvo en la aplicación de los mismos en el centro histórico de Camagüey; su evolución y actualización hasta el día de hoy en los planes vigentes, de la Dirección de Plan Maestro de la Oficina del Historiador de la ciudad es su principal logro. El diseño sistémico de las investigaciones del Centro de Estudios de Conservación de Centros Históricos y Patrimonio Edificado (CECONS) de la Universidad de Camagüey, a partir de este primer efecto, con la participación de profesores y estudiantes de arquitectura e ingeniería civil aportó un conjunto de resultados que se han aplicado también con éxito, en la práctica social.
\end{abstract}

Palabras clave: Centro histórico; conservación; métodos automatizados.

\section{Conservação de centros históricos. Métodos para estudos prévios.}

Resumo: O desígnio de algoritmos e métodos automatizados para obtenção da informação básica necessária para o planejamento urbano de um centro histórico foi na década dos 80's uma grande novidade científica cuja contribuição principal estava na aplicação do mesmo no centro histórico de Camagüey; sua evolução e atualização até o dia de hoje, em que o Plano Piloto do Escritório do Historiador da Cidade é sua realização principal. O desígnio sistêmico das pesquisas do Centro de Estudos de Conservação de Centros Históricos e Edifícios (CECONS) a partir desta primeira aplicação, com a participção de professores e estudantes de arquitetura e engenharia civil, contribuiu com outros resultados da Universidade de Camagüey, que foram aplicados com sucesso, na prática social.

Palavras-chave: Centro histórico; conservação; métodos automatizados.

\section{Conservation of historical centers. Methods for previous studies.}

Abstract: The design of algorithms and computer methods for obtaining of the necessary basic information for the urban planning of a historical center were in the decade of the 80's a great scientific novelty whose main contribution was in the application of the same ones in the historical center of Camagüey; its evolution and upgrade until today's day in the Partial Plan, Plan of Management and town-planning regulations currents, of the Master Plan of the Office of the Historian of the City are its main achievement. The systemic design of the researches of the Center of Studies of Conservation of Historical Centers and Hereditary Buildings (CECONS) of the University of Camagüey, starting from this first effect, with professors and students participation, it contributed with others results, that they have also been applied with success, in the social practice.

Keywords: Historical center; conservation; computer methods.

\section{(c) (1) $\Theta$}

DOI: https://doi.org/10.26512/patryter.v2i4.24975

Como citar este artigo: Gómez Consuegra, L. (2019). Conservación de centros históricos. Métodos para estudios previos. PatryTer - Revista Latinoamericana e Caribenha de Geografia e Humanidades, 2 (4), p. 18-27. DOI: https://doi.org/10.26512/patryter.v2i4.24975.

Recebido: 01 de julho, 2019. Aceite: 21 de agosto, 2019. Publicado: 01 de outubro, 2019.

${ }^{1}$ Arquitecta, Doctora en Ciencias Técnicas. Profesora Titular y Consultante de la Universidad de Camagüey, Cuba. ORCID: https://orcid.org/0000-0002-2187-647X . Email: lourdes.gomez@reduc.educ.cu 


\section{Introducción}

El centro histórico es parte integral de la ciudad y contenedor de lo mejor del patrimonio arquitectónico, urbano e intangible, núcleo fundacional de la misma, posee por una parte los valores que deben conservarse para las futuras generaciones y por otra las necesidades de modernización de la ciudad y de las nuevas funciones que deben formar parte de ella. Es por tanto un objeto de estudio del sistema patrimonial, de planeamiento, de medioambiente y de paisaje (Gómez, 2009b, pp.142-155).

La conservación de los elementos del patrimonio cultural debe estar fundamentada en estudios multidisciplinarios, de las áreas y edificios sometidos a diferentes categorías de intervención urbana y arquitectónica, de costumbres y tradiciones, en los que se consideren los factores socio-culturales y económicos, y donde exista un equilibrio entre lo tradicional y lo moderno. La conservación como actividad multidisciplinaria es aceptada por muchos con un carácter sistémico y holístico, que abarca el patrimonio tangible e intangible (Gómez\&Pérez, 2011, pp.260-275); pero sólo recientemente se comprenden sus interrelaciones en las diferentes escalas de trabajo, principalmente en la escala territorial.

El desarrollo de Planes Directores o Maestros de ciudades contemplan el estudio de los núcleos históricos como partes integrales de éstas, por tanto, es importante profundizar en los análisis, métodos y técnicas específicos, que permitan integrar el centro histórico como parte armónica de la ciudad (Gómez, 2010, pp.97-120). "Un centro histórico vivo obliga a compromisos para adecuarse a una circunstancia distinta de las de cuando se fue conformando y esos compromisos sucesivos aparecen evidenciados en las distintas capas del propio centro" (Coyula, 2008, p.60).

Sin embargo frecuentemente los planes se convierten en abstracciones utópicas, económicas, tecnológicas y de gestión. El enfoque de Paisaje Urbano Histórico (WHC/UNESCO, 2011) parece la forma más racional de acometer el problema en la actualidad, en el cual forma parte el planeamiento participativo (Gómez, 2012a). Otra forma de ver los planes es ir de las partes al todo considerando el centro histórico como una agregación de barrios - como fue tradicionalmente - o entidades casi autónomas donde los proyectos van conformando las propuestas (Gómez\&Pascual, 2007, pp.244-272), teniendo en cuenta los principios de conservación integral y preventiva, la incorporación de nueva arquitectura, la sostenibilidad y la participación comunitaria (Gómez, 2007, pp.46-54). Las transformaciones deben garantizar el arraigo de la población original, generando empleos dignos, viviendas adecuadas y espacios públicos que garanticen la convivencia y la vida ciudadana en estos espacios (Ziccardi et al 2015).

A partir del concepto del Coloquio de Quito (Gómez\& Peregrina, 2009, pp.307-309) se elaboró el siguiente concepto, del que se parte en la investigación:

\section{El centro histórico es la parte relativamente antigua de la ciudad que constituye un organismo integral de la misma; él posee el patrimonio cultural, la estructura física que identifica y refleja la evolución de un pueblo y su modo de vida, en las diferentes etapas de su formación (Gómez, 1986).}

La falta de instrumentos metodológicos sistematizados, en el ámbito internacional y Cuba, en la fecha en que se acometió la investigaciónsólo 3 años después del Coloquio de Quito- hizo que el objetivo general planteado fuera: estructurar un sistema de métodos que permitieran obtener la información básica necesaria para acometer el planeamiento urbano de un centro histórico; sobre la base del método científico general, de métodos específicos y la informatización del proceso; con la obtención de los resultados parciales derivados de su aplicación en el centro histórico de Camagüey, para su validación y propuestas.

Otros objetivos más específicos coadyuvarían a cumplimentar el objetivo general: la definición de los algoritmos para establecer el procedimiento para la elaboración del inventario general de edificaciones y sitios de valor y su procesamiento automatizado, así como de la delimitación precisa del centro histórico; las variables y relaciones para la determinación de las zonas tipológicas; las posibles estrategias de conservación y las bases para la restructuración del centro tradicional de la ciudad, en este caso, dentro del centro histórico.

Para acometer esta investigación, fue necesario incorporar a los estudiantes de arquitectura en diferentes formas de trabajo científico estudiantil, cuyos resultados parciales sirvieron de base a las investigaciones preliminares de la misma. Esta investigación produjo la primera tesis doctoral del grupo de investigaciones que luego se convertiría en el Centro de Estudios de Conservación de Centros Históricos y Patrimonio Edificado (CECONS), de la Universidad de Camagüey. Al comenzar la impartición de la Maestría en Conservación, las tesis de este nivel sirvieron para obtener resultados parciales $\mathrm{O}$ verificar y validar otros; mientras que las tesis de doctorado permitieron extender los resultados en otros ámbitos. 
Para calificar a los estudiantes participantes se ajustó el plan de estudios de la carrera de arquitectura con la impartición de un semestre en el cuarto año, dedicado a la conservación, en el que todas las asignaturas se integraban con el mismo objetivo, permitiendo la apropiación de los conocimientos y herramientas necesarias para las investigaciones del patrimonio y su conservación. (Gómez\& Alvarez, 2006)

Las líneas de investigación, primero del grupo y luego del CECONS fueron diseñadas y estructuradas como un sistema cuyos resultados se fueron integrando de forma armónica en resultados mayores y abarcadores, lo que permitió su aplicación inmediata en la práctica social, con lo que se obtuvieron dos reconocimientos importantes, entre otros muchos, en el 2008 la declaratoria de una zona del centro histórico de Camagüey como Patrimonio Cultural de la Humanidad (Gómez, 2009a) y tres Premios Nacionales de la Academia de Ciencias de Cuba, 2002, 2008 y 2011 (Gómez, et al., 2011).

El objeto de estudio de la investigación fue el centro histórico de Camagüey, declarado Monumento Nacional en 1980, (Fig. 1) el cual posee unas 330ha y 322 manzanas, que encierran 9241 edificaciones de las cuales unas 3450 presentan algún tipo de valor, en él residían 56000 habitantes aproximadamente. Del total de edificaciones inventariadas en 1984-86,el valor predominante fue el contextual, y un $38,7 \%$ poseía carácter excepcional, relevante o típico. El estado de la edificación no era crítico, el 88,2\% estaba entre regular y buen estado. El uso predominante era la vivienda con un $91 \%$. El estilo predominante, el ecléctico a pesar de ser conocida como una ciudad colonial; los edificios de una o dos plantas, con elementos característicos de la región central de la Isla: aleros de tornapunta, arcos, pilastras truncadas, techos de armadura y tinajones. Esta caracterización sigue siendo válida aunque algunos datos han variado, pero no significativamente.

El área declarada patrimonio Cultural de la Humanidad posee 54 ha y 80 manzanas, un total de 2843 inmuebles y en ella residen unos 8180 habitantes.

\section{Métodos para estudios previos en la conservación de centros históricos}

Las experiencias italianas en el estudio de los centros históricos en los años 70’s - propuestas metodológicas y acciones prácticas -, han sido paradigmáticas hasta nuestros días, pero mucho más en la época en que se elaboró la investigación
Figura 1 - El centro histórico de Camagüey, 1980 (contorno negro) y el área declarada Patrimonio Cultural de la Humanidad, 2008 (mancha roja)

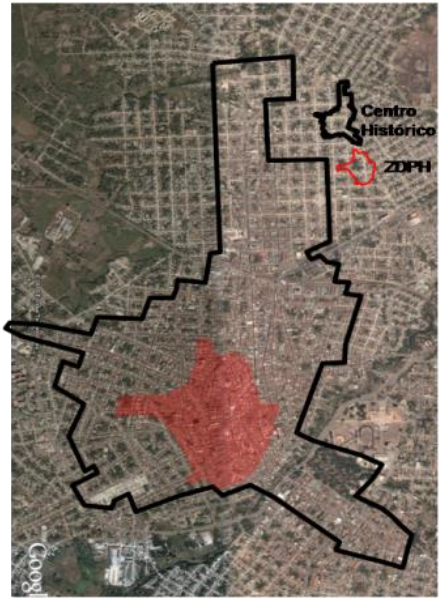

Fuente: Expediente de declaratoria de una parte del centro histórico de Camagüey, como Patrimonio

Cultural de la Humanidad (AAVV, 2008)

que se presenta, (1980-1986). Indispensables fueron para la fundamentación teórica de la misma los textos de Cevellati y Scannavini, Ciardini y Falini, Aldo Rossi, entre otros, de los cuales se hicieron eco algunos autores cubanos. Los casos de las ciudades de Bolonia y Ferrara constituyeron ejemplos muy estudiados.

Se plantearon cinco métodos con vistas a la obtención de las informaciones previas o preliminares para el planeamiento y la conservación de un centro histórico, mediante algoritmos fundamentados de forma teórica y práctica, la informatización del proceso y a su vez la interrelación entre los diferentes métodos. Se validaron los métodos y sus respectivos algoritmos y se obtuvieron resultados para el centro histórico de Camagüey.

Estos resultados se aplicaron de inmediato en los planes elaborados por las instancias institucionales dedicadas a la conservación del patrimonio, principalmente por la Oficina Técnica de Restauración de la Dirección Provincial de Patrimonio entre 1986-1997 con la que se trabajó en estrecha relación y a partir de 1998 por la Oficina del Historiador de la ciudad de Camagüey (OHCC).

\section{Estructuración del Inventario de edificaciones y sitios de un centro histórico}

El procedimiento científico se estableció después de realizar entre 1979 y 1984, el primer 
inventario y la primera actualización del mismo, a manera de "prueba piloto" y con la experiencia obtenida en el proceso de "prueba y error" mediante su perfeccionamiento paulatino.
Uno de los resultados automatizados más significativos de este método fue la definición de los Grados de Protección de los Edificios de acuerdo a la Ley cubana de protección del

Figura 2 - Algoritmo para la estructuración de los inventarios del patrimonio

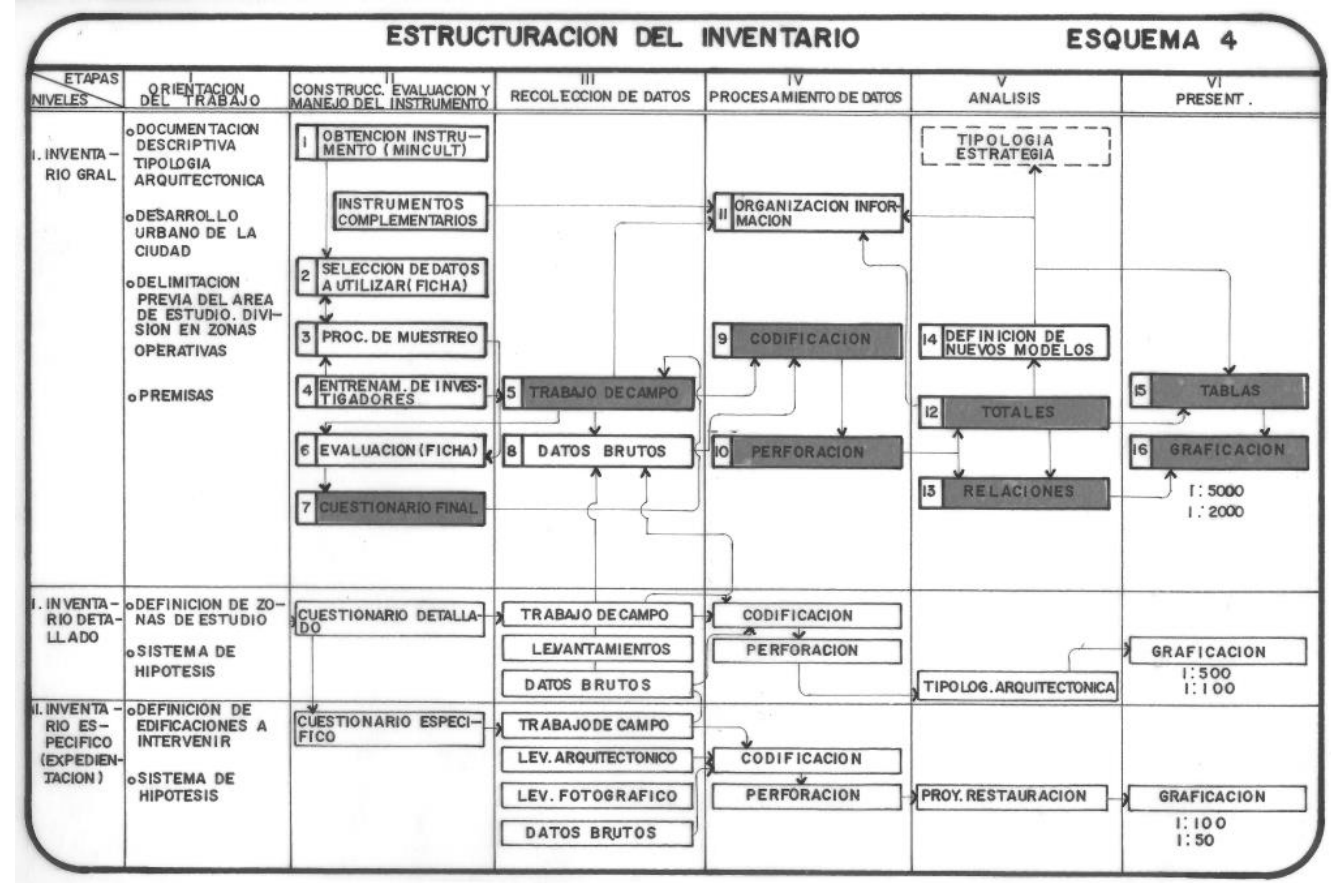

Fuente: Tesis doctoral Conservación de Centros Históricos (Gómez, 1986)

El algoritmo se estructuró en tres niveles: inventario general, para aplicar al universo estadístico o sea a todas las edificaciones y sitios del centro histórico; el inventario detallado o catálogo, para aplicar a las edificaciones o sitios detectados en el inventario general con algún tipo de valor o realizar alguna investigación detallada y los inventarios específicos o expedientación, para aplicar a las edificaciones o sitios a intervenir (Fig.2).

El inventario general se elaboró a partir de la ficha establecida por el Dirección Nacional de Patrimonio del Ministerio de Cultura de Cuba, pero ponderando las seis variables clave definidas como imprescindibles para precisar las características más importantes de las edificaciones (Ciardini \&Falini, 1983), con vistas a su conservación: valor, carácter, época, estado técnico, transformación y uso original, en ese orden de prioridad. Estas fichas se introdujeron en una base de datos, con la que pudo obtenerse no sólo los datos de uno, varios o grupos de edificios sino también, la cuantificación por manzanas, ejes, subzonas o zonas según se deseara la totalización y la consecuente caracterización de la unidad especificada así como el cruzamiento de variables con resultados más complejos, mediante la elaboración de programas de computación, que permitieron la toma de decisiones.

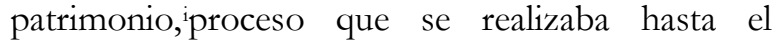
momento por apreciación de expertos. El inventario general fue la base informacional del resto de los métodos planteados facilitando los resultados obtenidos en éstos.

El inventario detallado se elaboró a partir de una ficha confeccionada al efecto (ficha de catálogo), en la que se especificaban en detalle los valores del edificio y en general las características que lo hacían único. (Gómez, 2000, pp.43-58) Mediante programas de computación se obtuvieron resultados detallados, como fueron: la caracterización tipológico-arquitectónica del centro histórico, las características estilísticas de los diferentes repertorios arquitectónicos, entre otros. En el caso de estudios o investigaciones detalladas, como pudieran ser acerca de los patios, los arcos, la carpintería en determinado período, entre otros muchos, habría que confeccionar fichas detalladas según el estudio deseado.

El inventario específico o expediente, constituido por "el proyecto de conservación", contenía los estudios históricos arquitectónico y urbano del edificio a intervenir, la o las categorías y tipos de intervención a las que estaría sometido, entre otras muchas decisiones a tomar en el proyecto, en dependencia de las características, 
valores, singularidad, estado técnico, entre otros aspectos del edificio.

\section{Determinación de las zonas tipológicas urbanas}

La conservación del patrimonio debe evitar a toda costa la alteración de las tipologías tanto a escala arquitectónica como urbana; y su estudio ofrece un testimonio insustituible de los procesos creativos que han dado lugar a las formas que hoy se presentan a nuestro uso y percepción. Sólo el conocimiento profundo y respeto de las tipologías y las estratigrafías urbana y arquitectónica heredadas, permitirá la readecuación o reconfiguración, tanto del tejido urbano como de los edificios a las nuevas necesidades sociales.

La tipología urbana es el resultado de la agrupación de las manzanas de una zona urbana en tipos o modelos urbanos a partir de la determinación de rasgos comunes, que las hacen integrar un conjunto claramente identificable y homogéneo, en virtud de su morfología, características ambientales, históricas y socioeconómicas.

Las variables definidas en el algoritmo para el estudio de la morfología urbana y con ello la determinación de las zonas tipológicas fueron: manzana (forma y tamaño), lote o parcela (forma, tamaño y asociación), sección vial y alturas, cronología de ocupación, valor, y espacios abiertos. Se procedió a un análisis de fotointerpretación para la obtención de los datos y su posible automatización posterior.

A partir de la elaboración de una base de datos por manzana, con las nuevas variables, complementadas por las del inventario general, como el valor, carácter y ocupación de la manzana, se estableció la relación entre forma de la manzanademás variables, y mediante un programa computacional de predominios, se establecieron las manzanas cuyas características son comunes y conforman una zona. (Gómez \& Gamboa, 1986, pp.40-45) Este método permitió la caracterización tipológico-urbana general del centro histórico.

Para el caso del centro histórico de Camagüey, la misma fue de predominio de manzanas irregulares con lados curvosii ${ }^{\mathrm{i}} \mathrm{y}$ medianas; lotes irregulares, medianos-pequeños, asociados por pared medianera y pasillo lateral; sección vial estrecha de acera y calle; altura de una planta; cronología de ocupación del siglo XIX; valor medio-bajo y espacios abiertos reducidos, aunque al interior de las manzanas con verde abundante en las zonas residenciales.

Cuatro tipos urbanos principales fueron establecidos: Tipo I, manzanas irregulares con lados curvos de alta centralidad y altos valores; Tipo I subtipo 2, manzanas irregulares con lados curvos de baja centralidad y altos valores; Tipo II, manzanas poligonales medianas, residenciales, y de bajo valor; Tipo III, manzanas rectangulares o cuadradas, residenciales (Vigía y La Caridad); Tipo IV, manzanas poligonales grandes, residenciales, de valor medio. Estos cuatro tipos urbanos quedaron distribuidos en diez zonas tipológicas, dentro de las que se incluye el parque urbano Casino Campestre, que es atípica (Fig. 3).

La graficación de las zonas tipológicas en el plano del centro histórico de Camagüey se realizó de forma manual ya que los S.I.G. no estaban desarrollados suficientemente en esa época en Cuba, pero este resultado tuvo amplia repercusión y uso para la toma de decisiones de conservación y sigue siendo válido en la actualidad.

Figura 3 - Plano de Zonas tipológico-urbanas. Los cuatro tipos urbanos definidos se distribuyen en diez zonas homogéneas

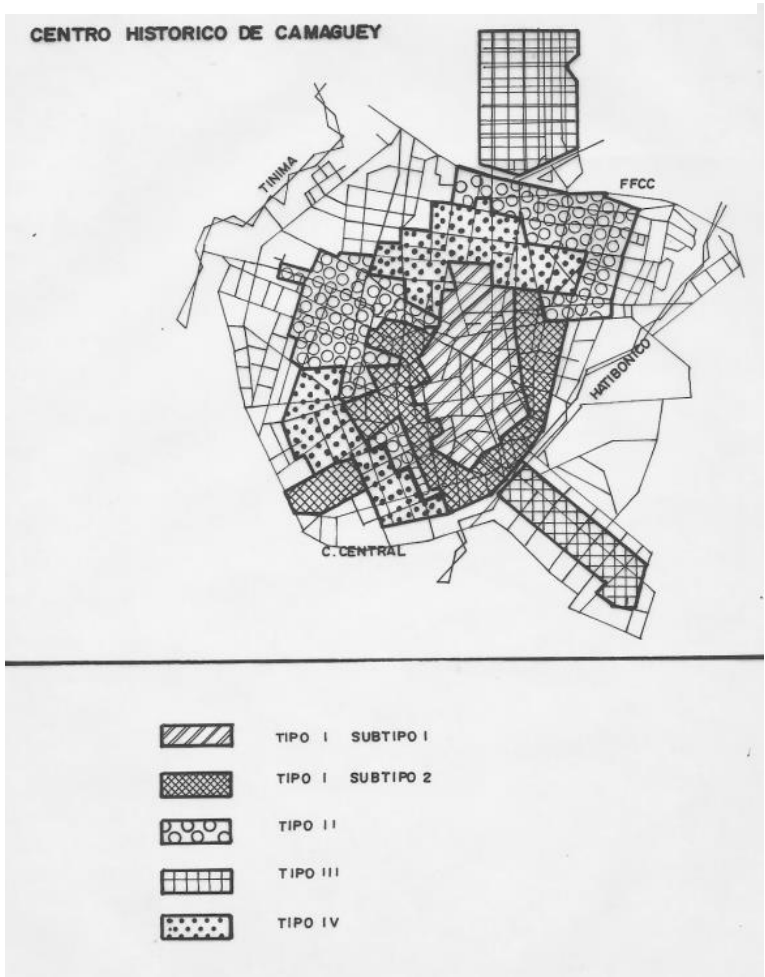

Fuente: Tesis doctoral Conservación de Centros Históricos (Gómez, 1986)

\section{Estrategia de conservación}

La necesidad de establecer en el marco de los estudios generales para el planeamiento urbano de un centro histórico, las prioridades de intervención, constituye un aspecto central, por lo que en la investigación se estableció un algoritmo para la elaboración de las estrategias de intervención, entendidas como las prioridades que se establecen para acometer los trabajos de 
conservación según la atención a determinada variable o grupo de variables y se pudo establecer la de carácter puntual o sea el orden en que debe intervenirse los edificios según su importancia, valores y características; por manzana, por eje, por zonas o subzonas, según los intereses de conservación, los tiempos disponibles y la políticas seguidas (Ciardini \& Falini, 1978).

Se partió del principio que es imposible acometer la conservación total de un centro histórico en un corto plazo "hay que dilucidar qué se puede o se debe conservar, renovar, reconvertir para un nuevo uso o directamente derribar para fabricar un nuevo espacio urbano" (Borja, Herce\&Muñoz, 2010, p.32). De ahí la necesidad de conocer aquellas edificaciones y zonas de mayor valor y que por su ubicación en la ciudad, su carácter excepcional o relevante o el estado técnico en que se encontraban, deberían ser las primeras en recibir acciones de conservación.

La estrategia puntual o por edificio se estableció directamente de los datos del inventario general con procedimientos sencillos de ordenamiento computacional. La estrategia por manzana, eje o zona y subzona implicó la introducción en una nueva base de datos de variables específicas. En el caso de las manzanas y zonas o subzonas debió conocerse el área de las manzanas y el índice de centralidad de cada una y calcular el \% de valor y la densidad de valor de la manzana; mientras que para los ejes, la longitud del mismo, el índice de centralidad, el cálculo dela densidad y el \% de valor lineal (Gómez, 1986).

Fueron definidas las zonas más importantes o jerarquizadas del centro histórico y dentro de ellas, las manzanas, ejes o edificaciones más importantes o significativas. Estos resultados sólo pudieron obtenerse con la automatización del proceso cuya instrumentación manual es casi inoperable. Con estos resultados se pudieron conformar etapas de ejecución en dependencia de la disponibilidad de recursos, tiempos disponibles y proyectos elaborados (Cervellati, 1978). Se obtuvo un esquema de la estructura de valor que debía revalorizarse en el centro histórico de Camagüey con vistas a la conservación de lo más valioso del mismo. Esta estructura estuvo en función de los recorridos de visitantes nacionales y extranjeros. (ver Fig.4)

\section{Restructuración del centro de la ciudad}

La coincidencia del centro de la ciudad en el centro histórico es una de las formas en que más se evidencia la contradicción conservación vs. renovación, implícita en el proceso de protección del patrimonio cultural; por una parte la necesidad de conservación al máximo de los valores heredados y por la otra la solución a las crecientes necesidades de una sociedad contemporánea, además de la imposibilidad de crecimiento del centro de ciudad sin producir un proceso de terciarización o gentrificación fuera de escala. Es necesario por tanto, establecer un verdadero equilibrio, al tener en cuenta las actividades que pueden desarrollarse en ese entorno y aquellas que resultan conflictivas y dañinas y deben sacarse de él: "las actividades han de admitir una necesaria flexibilidad de forma y concepto que las capacite para ser una de las claves del futuro urbano". (Barnada et al., 2010, p.17)

En el caso de Camagüey el centro de la ciudad se encuentra en pleno centro histórico, de ahí que a la hora de estudiar todos los aspectos que intervienen en la conservación del mismo, se debe tener en cuenta esta importante y vital zona de la ciudad y su posible restructuración, mediante su organización funcional y no de una zonificación que era la práctica frecuente. El centro de la ciudad de Camagüey presenta un desarrollo lineal. La irregularidad de la trama así como la estrechez de las calles introduce un problema adicional de vialidad. El conflicto vehículo peatón estuvo presente en esta zona, por dónde circulaba el transporte público. Uno de los resultados obtenidos de este estudio fue la recomendación de peatonalización de ejes principales.

El algoritmo elaborado tuvo en cuenta la caracterización del centro de la ciudad, los polos de actividades en que se estructura el mismo y la jerarquía de funciones. Se elaboraron matrices ponderadas de las actividades existentes yen déficit, para lograr la reanimación de algunas zonas deprimidas, el balance diurno y nocturno y la compatibilidad de otras actividades que debían eliminarse - industrias, taller, ciertas oficinas, entre otras.

Para el posible cambio de funciones se tuvieron en cuenta dos variables clave: el área disponible en contraposición con el área necesaria y la tipología arquitectónica (Rossi, 1971). La clasificación tipológico-arquitectónica del centro histórico de Camagüey, se elaboró mediante la definición de las variables que intervienen en ese proceso, mediante una tesis de maestría (Sariol, 1984) basada en la clasificación establecida para Bolonia con su adaptaciones locales, (Cervellati \& Scanavini, 1976) y cuyo procesamiento automatizado logró gran precisión. Se elaboraron programas de computación para llegar a variantes de propuestas de restructuración de acuerdo a los diferentes escenarios que podrían presentarse. 


\section{Delimitación del centro histórico}

La delimitación de un centro histórico no puede definirse con precisión sólo por los valores que él contiene, por simple inspección; es necesario hacer intervenir otras variables que permitan tener en cuenta los factores determinantes en el problema. Previamente y utilizando los métodos manuales a manera de "prueba piloto" se desarrolló un primer acercamiento al problema(Machado, 1985).

Para la elaboración del inventario general se estableció una amplia zona, mayor que la definida por el Plan Director de la ciudad de Camagüey y que sería evaluada, mediante el cálculo de los indicadores por manzanas y ejes planteados en el método de tipología urbana como: el porcentaje de valor, la densidad de valor, y el índice de centralidad, con lo que se fueron precisando los límites de valor más restringidos. Para ello se tuvo en cuenta además de los resultados del inventario general, los de tipología urbana, la restructuración del centro de la ciudad, la estrategia de conservación, así como el desarrollo histórico de la zona.

Con el cruzamiento de estos resultados se obtuvo otro que resulta muy importante, la definición de Zonas de Protección o zonas homogéneas que se convertirían posteriormente en sectores de regulación urbanística.

Aplicaciones e introducción de resultados en la práctica social

La aplicación de los cinco métodos en el centro histórico de Camagüey a manera de validación de los resultados obtenidos, conllevó la elaboración del Anteproyecto de Plan Director del centro histórico de Camagüey (Aguilera \&Gómez, 1984, pp.56-61)y más tarde el Plan Director (Gómez,1985, pp.43-49) que sirvieron de base a la Oficina Técnica de Restauración, institución dependiente de la Dirección Provincial de Patrimonio y responsable de llevar adelante los trabajos de conservación, en aquella época.

Los resultados obtenidos para el centro histórico de Camagüey fueron en su mayoría de carácter urbano por lo que se evidenció la necesidad de nuevas investigaciones para los estudios de tipologías arquitectónicas, del repertorio colonial (Prieto, 1991), ecléctico (Mas, 2000) y religioso (Echemendía, 1998); así como de la historia de la ciudad (Chaos,2005), elaborados por especialistas del Grupo de Investigación, con sendas tesis doctorales, indispensables para el perfeccionamiento de las valoraciones realizadas de la arquitectura camagüeyana. Ello derivó en un libro que aborda el análisis histórico de la ciudad y sus edificios (Gómez, 2006).
Al crearse la Oficina del Historiador de la ciudad de Camagüey en 1998, los resultados de la investigación fueron incluidos por la Dirección de Plan Maestro de dicha oficina, actualizados posteriormente sobre la base de estos antecedentes pero con nuevos enfoques metodológicos como es el de sostenibilidad y el de Paisaje Urbano Histórico (PUH), siempre bajo la dirección de esta autora.

Estos resultados se socializaron internacionalmente en un libro sobre la Conservación de los Centros Históricos en Cuba (Gómez, 2015).

Estructuración del Inventario de edificaciones y sitios de un centro bistórico

Los inventarios del centro histórico de Camagüey fueron actualizados, en los años 1990 para el Equipo Técnico de Restauración (Montero, 1990), y en 1997-99, 2005-06 y 2010, para la Dirección de Plan Maestro de la Oficina del Historiador de la ciudad de Camagüey (OHCC), con la asesoría de esta autora y se elaboró también un multimedia a manera de instructivo para el uso y explotación de los datos del inventario general.

Para la actualización de 1999 se elaboró una aplicación por parte de GeoCubaii con el objetivo de facilitar las consultas y actualización del mismo, mediante la cual cada especialista de la Dirección de Plan Maestro desde su puesto de trabajo podía consultar y actualizar los datos de los inventarios.

La actualización realizada en 2005-06, tuvo tres objetivos principales: la elaboración del Plan Parcial del 2006(AAVV, 2006)(Gómez\& Pascual, 2013, pp.47-59), el Plan de Manejo del 2007 (AAVV, 2007), para lo que se diseñó una metodología de carácter híbrido — planeamiento tradicional y estratégico-, en base a las experiencia anteriores; así como la confección del expediente de declaratoria de una parte del centro histórico de Camagüey como Patrimonio Cultural de la Humanidad (ZDPH), (AAVV, 2008) aprobado en Quebec en 2008 (WHC/UNESCO, 2008)iv.

Un estudio realizado a partir del inventario general, permitió el conocimiento detallado de los edificios Grado de Protección I del Área declarada Patrimonio de la Humanidad (ZDPH)(Caballero, 2008) y crear un catálogo digital de los mismos.

La actualización realizada en el año 2010 se realizó para la reajuste de los planes antes mencionados, (cada 5 años) y que se actualizaron por cada una de las variables definidas en el Plan Parcial: el turismo en la zona Patrimonio Cultural de la Humanidad de Camagüey, (2014-2015); (Gómezet al., 2015) el medioambiente en la zona Patrimonio Cultural de la Humanidad de Camagüey, (2015-2016) (Gómez et al., 2016); 
impactos de la actividad por cuenta propia en el Paisaje urbano histórico de Camagüey, (2016-2017) (Gómez et al., 2017) y el paisaje urbano histórico en el Plan Parcial de la zona Patrimonio Cultural de la Humanidad de Camagüey, (2017-2018) (Gómez et al., 2019). Además de la redacción de las Regulaciones urbanísticas para el centro histórico de Camagüey que se publicaron en forma de libro (AAVV, 2012).

La experiencia fue socializada internacionalmente, en eventos científicos pero principalmente en cursos impartidos en diferentes universidades e instituciones: diez mexicanas (Yanez, 2006); una brasileña, dos ecuatorianas, una argentina y cuatro españolas.

Con la experiencia acumulada se extendieron los resultados, bajo la codirección de esta autora, en el centro histórico de la ciudad de Puebla en 1994 con vistas a la elaboración del Plan Parcial bajo la dirección del arquitecto mexicano Dr. Nicolás López Tamayo, y con la asesoría del arquitecto Giorgio Lombardi, de Italia (Gómez, López\&Fuentes, 1995).

Años más tarde, y con una visión mucho más amplia del patrimonio de una región, se elabora por un arquitecto mexicano, una tesis doctoral cuyo objeto de estudio fue el Valle de Ameca, Jalisco, en la cual el diseño de fichas específicas para los diferentes tipos de patrimonio existentes en ese caso y la elaboración de su inventario general tuvo gran importancia. (Guerrero, 2009).

Se elaboró, en base del catálogo, la Guía de Arquitectura y Paisaje de las provincias de Camagüey y Ciego de Ávila, publicada por la Junta de Andalucía (Gomez et al., 2009) y en el nivel de inventario específico, se acometió el estudio detallado de algunos edificios importantes, como el edificio más antiguo de Camagüey, el antiguo convento de San Francisco(Gómez \& Puente, 2015, pp.89-107).

\section{Determinación de las zonas tipológicas urbanas}

Los resultados obtenidos en el estudio de la tipología urbana del centro histórico de Camagüey resultan válidos hasta el día de hoy. El plano resultante no pudo elaborarse de forma automatizada, por las razones explicadas; sin embargo para el caso de la ciudad de Puebla, México, los planos, incluyendo los del inventario se elaboraron en ArcView.

Otros trabajos complementaron estos resultados como el análisis de la estructura simbólica del centro histórico de Camagüey y de la imagen urbana (Abramova, 2002) así como de la conservación de la trama urbana de Camagüey (Bermúdez, 2010).

\section{Estrategia de conservación}

Los resultados obtenidos en este aspecto, principalmente con fines turísticos fue complementado más tarde con una tesis de maestría. (Plá, 2002)

Este método también se aplicó en el centro histórico de Puebla, México, (Gómez, López, \& Fuentes, 1995) lo que demostró su validez para cualquier centro histórico, siempre y cuando se tenga realizado el inventario general o se elabore previamente.

Con relación a las medidas relacionadas con el medioambiente se realizaron trabajos posteriores que estudiaron la contaminación ambiental en el centro histórico de Camagüey (Avilés, 1994) - tesis doctoral -, la posible aplicación de los criterios de sostenibilidad presentes en el Programa Agenda 21 (Guzmán, 2005); los estudios generales medioambientales de ejes urbanos principales (Varona, 2005) y su conexión a los nuevos centros urbanos(Almeida \& Santana, 2011), como tesis de maestría.

$\mathrm{Al}$ actualizar los planes vigentes con un nuevo enfoque de PUH se acometieron trabajos que conceptualizan esta nueva forma de ver la ciudad histórica (Gómez, 2012b, pp.1109-1120) y su aplicación en el centro histórico de Camagüey (Gómez,2018, pp.88-107), así como aspectos prácticos como es la inserción de nueva arquitectura en parcelas edificables(Almeida, 2013) y la protección de vistas relevantes.(Almeida \&Torrens, 2017) Otros estudios teórico-prácticos fueron llevados a cabo, sobre la relación morfotipológica y el bioclima térmico(Rodríguez, 2016) y (Rodríguez et al., 2016, pp.85-101).

\section{Conservación de la vivienda en el centro histórico de Camagüey}

Un acápite importante dentro de la Estrategia de Conservación se desarrolló sobre la problemática de la vivienda desde las escalas más generales de la ciudad (Marichal, 2005), el centro histórico y la recuperación de su fondo habitable (Reyes, 1986), hasta estudios de barrios como el de San Juan de Dios en el que se realizó un proyecto comunitario internacional ${ }^{v}$ que incluía entre otros aspectos un plan urbano del barrio (Pintado, 2002), su estrategia de rehabilitación integral urbana (Betancourt, 2004) y (Rodríguez, 2005) y arquitectónica (Kaba, 2005), así como la formación de escolares del barrio. Se estudiaron las transformaciones más comunes que afectaban las viviendas, en un análisis comparativo de más de 20 
años (García, 1994) y (Novoa, 2015) y cómo las intervenciones en cuarterías ${ }^{\mathrm{vi}}$ pudieran encaminarse correctamente(Alonso, 2010) con el fin de favorecer la solución del problema más acuciante del centro histórico de Camagüey, dónde la mayoría de sus edificaciones son viviendas (91\%).

Estos resultados pudieron extenderse en la Rehabilitación de la vivienda de una ciudad gallega, Mondoñedo, plan llevado adelante por el ayuntamiento de esa ciudad entre 1998-2000, con la asesoría de esta autora (Gómez, 1998-2000). Para esa ciudad se realizaron proyectos urbanoarquitectónicos, uno de ellos ejecutado (Gómez, 2005, pp.66-73).

\section{Restructuración del centro de la ciudad}

Los resultados en este aspecto fueron concretándose paulatinamente, inicialmente con proyectos de reanimación urbana y eliminación del transporte público en los ejes principales. La peatonalización y refuncionalización de los ejes comerciales fueron recomendados; sin embargo a partir de los estudios elaborados para el Plan Parcial de 2006 hubo que realizar algunos ajustes, en cuanto a las restricciones para los cambios de uso, por lo que fue necesario la actualización del método (Lugones, 2008) siendo el que se aplica a partir de esa fecha. La peatonalización en 2011 de la calle Maceo fue un ejemplo paradigmático de conservación integral, el "paseo peatonal calle Maceo" (ver Fig. 4b), que se multiplicó en otros ejes principales (Pascual \&Gómez, 2014, pp.244272). En el año 2014, la peatonalización total de la "calle República", netamente comercial y de 1,5 km de longitud, con etapas previas de vía de convivencia, solo para ciclos, entre otras; el "paseo temático del cine", como calle de convivencia y recientemente en 2019 el "paseo del ferrocarril", a continuación de la calle República. Otros resultados derivados de la aplicación del método consisten en la elaboración de normativas para la conservación de la imagen en la zona comercial del centro histórico de Camagüey (González, 2002), y las normativas para los espacios públicos (Marín, 2005).

\section{Delimitación del centro bistórico}

Con la información del inventario general elaborado en 1984, se realizó en 1988 el expediente de delimitación del centro histórico de Camagüey de la Dirección Provincial y Nacional de Patrimonio, para su actualización oficial y declaratoria de Monumento Nacional.

En el año 2010, la Dirección de Plan Maestro de la Oficina del Historiador de la ciudad de Camagüey, cuestionó la delimitación oficial del centro histórico de Camagüey establecida en 1988 y acomete su actualización mediante una tesis de maestría tutoreada por esta autora (Pérez, 2010). Los resultados ratificaron las sospechas iniciales, la pérdida de valores sufrida en la periferia del centro histórico, lo que provocó el corrimiento del límite hacia adentro y la consiguiente pérdida de protección de algunas áreas.

A partir del año 2015 se ha comenzado la nueva verificación del inventario general extendido a todo el centro histórico de la ciudad de Camagüey, aún en proceso y con ello lograr precisar el límite obtenido en el estudio anterior; ya que esta área además de estar declarada como Monumento Nacional desde 1980, constituye el área de amortiguamiento de la zona declarada Patrimonio Cultural de la Humanidad (ZDPH).

Las zonas de protección establecidas en la investigación estuvieron vigentes hasta el 2006, cuando fueron incluidas y llevadas a una escala de más detalle en el Plan Parcial de la ZDPHy su Plan de Manejo, 2007 y aún más cuando se redactaron las regulaciones urbanísticas en 2008-12. (AAVV,

Figura 4 -Dos conjuntos urbanos fueron sometidos en momentos diferentes a rehabilitación urbana y conservación integral respectivamente. La Plaza de San Juan de Dios, la más emblemática del centro histórico de Camagüey, 1987, actualizada hoy, y el paseo Peatonal “calle Maceo”, 2011

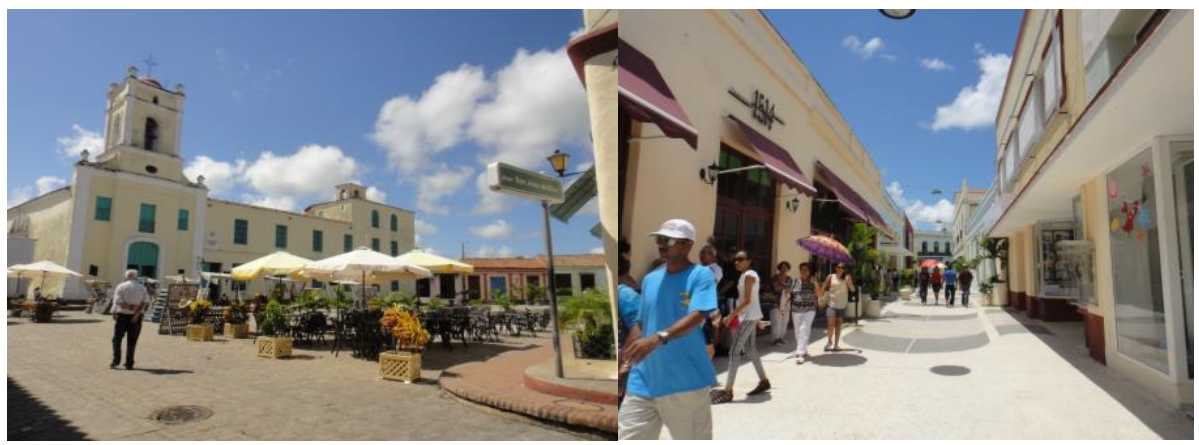

Fuente: Fotos de la autora 
En esa fecha, al realizar la actualización del Plan Parcial (cada 5 años) las dos zonas de protección definidas inicialmente para el centro histórico de Camagüey, como zonas I y II, fueron a su vez detalladas para la ZDPH, las que se convirtieron en 4subzonas de protección o regulación urbanística y 7 áreas homogéneas. (Fig.5) conservación y la delimitación precisa del centro histórico-y la aplicación de procesos automatizados en los mismos, usados por primera vez en Cuba.

En los resultados obtenidos inicialmente y luego en su enriquecimiento paulatino en el tiempo, tuvo gran importancia la participación de estudiantes e investigadores del CECONS, de la Universidad de Camagüey, quienes con trabajos

Figura 5 - Las dos zonas de protección definidas inicialmente en 1986, a partir de las zonas de Tipología Urbana I subtipo 1 y 2 , al ser estudiadas en detalle en la ZDPH, se convierten en cuatro subzonas y siete áreas homogéneas más específicas, como sectores de regulación urbanística, 2012. (AAVV, 2012, p.198)

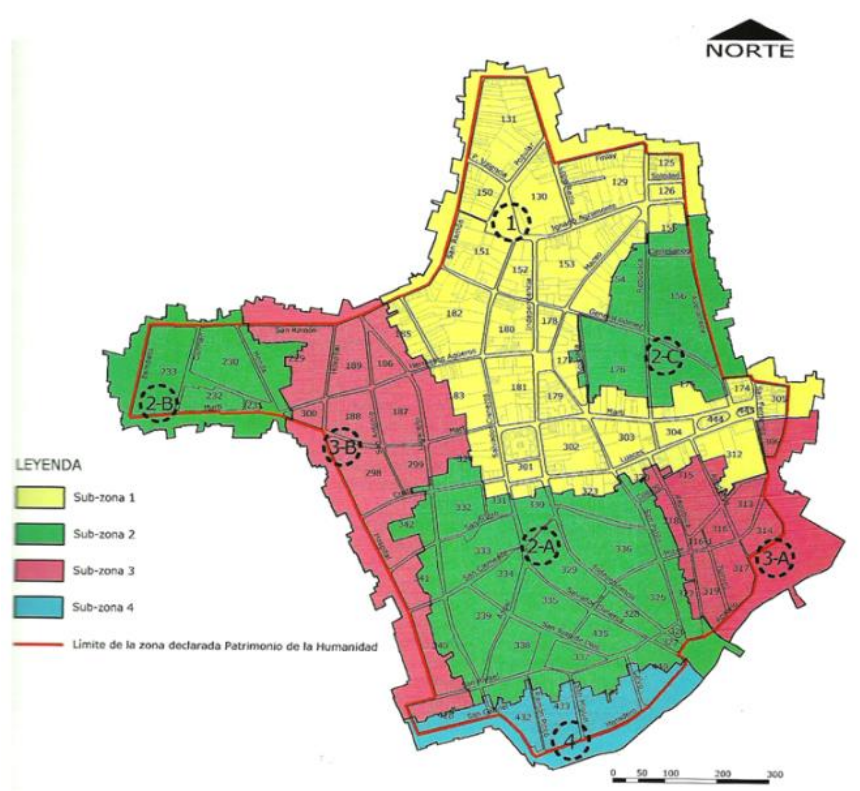

Fuente: Regulaciones urbanísticas del centro histórico de Camagüey

\section{Conclusiones}

La investigación presentada, aunque fue desarrollada en un lapso de seis años (1980-86), sus resultados han repercutido hasta la actualidad en sucesivas verificaciones, actualizaciones y aplicaciones, en los que siempre ha participado esta autora. Una metodología de carácter híbrido, con criterios de sostenibilidad, participación ciudadana y conservación integral fue concebida para la elaboración de los Planes Urbanos vigentes (200607 ), a la que se fueron incorporando nuevos enfoques metodológicos como el de Paisaje Urbano Histórico.

En su momento los aportes principales que le otorgaron originalidad a la investigación fueron: la sistematización mediante algoritmos de cinco métodos que permiten obtener las informaciones básicas para la toma de decisiones en la conservación del centro histórico de Camagüey los inventarios general, detallado y específico, las zonas tipológicas y de protección, la definición de una estructura de valor como primera fase de docentes, de diploma, tesis de maestría y doctorado, planificados en el cumplimiento de un objetivo común, aportaron innumerables resultados, incorporados en cuarenta años de evolución, en las instituciones que deciden en la conservación del patrimonio, especialmente en la OHCC.

Con la aplicación de los resultados obtenidos en el centro histórico de Camagüey y la experiencia adquirida, no solo se generalizaron los mismos a otros centros históricos de Cuba, sino que pudieron extenderse en el ámbito internacional, como en el centro histórico de la ciudad de Puebla, México y el de Mondoñedo, España; así como a otros países mediante la impartición de cursos de postgrado, maestría y doctorado.

\section{Referencias Bibliográficas}

AAVV. (2006). Plan Parcial de la zona declarada Patrimonio de la Humanidad (ZDPH) del centro 
bistórico de Camagüey. Dirección de Plan Maestro, OHCC, Camagüey.

AAVV. (2007). Plan de Manejo de la zona declarada Patrimonio de la Humanidad (ZDPH) del centro bistórico de Camagüey. Dirección de Plan Maestro, OHCC, Camagüey.

AAVV. (2008). Expediente de declaratoria de una zona del centro histórico de Camagüey. CamagüeyQuebec.

AAVV. (2012). Regulaciones urbanísticas. Ciudad de Camagüey. Centro Histórico. Camagüey: Ediciones El Lugareño.

Abramova, I. (2002). Caracterización tipológica de la imagen urbana actual del centro histórico de Camagüey. Tesis de maestría . Universidad de Camagüey, Camagüey, Cuba.

Aguilera, R. M., \& Gómez, L. (1984). Anteproyecto de Plan Director del Centro Histórico de Camagüey. Arquitectura y Urbanismo, $V$ (2), 56-61.

Almeida, M., \& Santana, A. (2011). Propuesta de conexión vial del centro histórico y el centro político-administrativo de la ciudad de Camagüey. Trabajo de diploma . Universidad de Camagüey, Camagüey, Cuba.

Almeida Torrens, M. (2013). Inserción urbanoarquitectónica en parcelas edificables del centro histórico de la ciudad de Camagüey. Tesis de maestría. Universidad de Camagüey, Camagüey, Cuba.

Almeida, M., \& Torrens, M. (2017). Impactos en el Paisaje Urbano Histórico en la Ciudad de Camagüey. Análisis de las Vistas Relevantes en torno a los Hitos principales. XI Simposio Desafios en el Manejo y Gestión de Ciudades. Camagüey.

Alonso Miranda, L. (2010). Criterios generales para la Intervención de cuarterías de la Zona Priorizada del Centro Histórico de Camagüey. Tesis de maestría. Universidad de Camagüey, Camagüey, Cuba.

Avilés Merens, D. (1994). Contaminación ambiental en el centro histórico de Camagüey. Tesis doctoral . Instituto Superior Politécnico "José A. Echeverría". ISPJAE, La Habana, Cuba.

Barnada, J., \& otros. (2010). Rehabilitación y reconversión de los tejidos urbanos. Estrategias e instrumentos. Curso Rebabilitación de los centros históricos y barrios degradados. Barcelona: Universitat Oberta de Catalunya.

Betancourt, E. (2004). Estrategia para la rehabilitación integral del barrio San Juan de Dios. Trabajo de diploma. Universidad de Camagüey, Camagüey, Cuba.

Bermúdez, D. (2010). Conservación de la trama urbana del centro histórico de Camagüey.
Tesis de maestría. Universidad de Camagüey, Camagüey, Cuba.

Borja, J., Herce, M., \& Muñoz, F. (2010). Ciudad y espacio público. Curso Rahabilitación de centros históricos y barrios degradados. Barcelona: Universitat Oberta de Catalunya.

Caballero, Y. (2008). Estudio de los edificios de grado de protección 1 ubicados en la zona declarada patrimonio cultural de la humanidad del centro histórico de Camagüey. Tesis de maestría. Universidad de Camagüey, Camagüey, Cuba.

Cervellati, P. L. (1978). El proyecto de la conservación. En AAVV, Los centros históricos. Politica urbanistica y programa de la actuación. (págs. 103-113). Barcelona: Gustavo Gili.

Cervellati, P. L., \& Scanavini, R. (1976). Bolonia. Politica y Metodología de Restauración de los Centros Históricos. México DF.: Gustavo Gili.

Chaos Yeras, M. (2005). Lenguaje de poderes en la estructura física de Santa María del Puerto del Príncipe. Siglos XVI-XVIII. Tesis doctoral . Universidad Pablo Olavide, Sevilla, España.

Ciardini, F., \& Falini, P. (1978). La actuación pública en los centros históricos. En AAVV, Los centros bistóricos. Política urbanistica y programa de la actuación. (págs. 115-267). Barcelona: Gustavo Gili.

Ciardini, F., \& Falini, P. (1983). Los Centros Históricos. Barcelona: Gustavo Gili.

Coloquio de Quito. (2009). En L. Gómez, \& A. Peregrina (comp. y coord.), Documentos internacionales de conservación y restauración (págs. 307-309). Guadalajara: Instituto Nacional de Antropología e Historia-CONACULTA.

Coyula Cowley, M. (2008). Los muchos centros de La Habana. En AAVV, VII Encuentro Internacional Renovación de centros históricos. La arquitectura de hoy, entre la ciudad histórica y la moderna. México DF: Centro Cultural de España en México.

Echemendía Morffi, A. (1998). Caracterización de los templos coloniales del Centro Histórico de Camagüey. Tesis doctoral . Instituto Superior Politécnico "José A. Echeverría" ISPJAE, La Habana, Cuba.

García Fernández, E. (1994). La renovación. Análisis arquitectónico en el centro histórico - Trabajo de diploma . Universidad de Camagüey, Camagüey, Cuba.

González Bedoya, L. (2002). Normativas para la conservación de la imagen en la zona comercial del centro histórico de Camagüey. Tesis de maestría. Universidad de Camagüey, Camagüey, Cuba.

Gómez Consuegra, L. (1985). Plan Director del Centro Histórico de Camagüey. Arquitectura Cuba, XXXV (361-362), 43-49. 
Gómez Consuegra, L. (1986). Conservación de Centros Históricos. Tesis doctoral . Instituto Superior Politécnico "José A. Echeverría". ISPJAE, La Habana, Cuba.

Gómez Consuegra, L. (1998-2000). MemoriaPrograma de Rehabilitación da Área de Rehabilitación declarada do Casco e dos Barrios Históricos de Mondoñedo. Concello de Mondoñedo, Mondoñedo, Galicia, España.

Gómez Consuegra, L. (2000). El valor arquitectónico y urbano en las estrategias de intervención. La ciudad como proyecto. Estrategias patrimoniales (págs. 43-58). Ribadeo, Lugo, España: Diputación provincial de Lugo.

Gómez Consuegra, L. (2005). Un proyecto para Mondoñedo. Parque Urbano AlamedaCementerio. Arquitectura y Urbanismo, XXVI (2), 66-73.

Gómez Consuegra, L. (2006). El centro histórico de Camagüey. En L. Gómez, O. Prieto, \& V. Mas, Camagüey, Ciudad y Arquitectura (15141950) (págs. 15-84). Camagüey, Cuba: Ácana.

Gómez Consuegra, L. (2007). Sociedad y Universidad; participación comunitaria consciente en la conservación del patrimonio. Experiencias en el barrio San Juan de Dios en el Centro Histórico de Camagüey, Cuba. PRAGMA, O (00 edición especial), 46-54.

Gómez Consuegra, L. (2009). El Centro Histórico Urbano de Camagüey, Patrimonio Mundial. Planeamiento, Gestión y Manejo. Apuntes, 22 (2), 142-155.

Gómez Consuegra, L. (2009). El Centro Histórico de Camagüey. Patrimonio Cultural de la Humanidad. En E. Cento (coord.), Cuadernos de Historia Principeña No.8 (págs. 11-22). Camagüey, Cuba: Ácana.

Gómez Consuegra, L. (2010). Políticas urbanas para la conservación del centro histórico. $L a$ reinvención de la Metrópoli. Temas de estudio (págs. 97-120). Guadalajara, México: El Colegio de Jalisco.

Gómez Consuegra, L. (2012). Planeamiento participativo para la conservación de un barrio del centro histórico de Camagüey, Cuba. En E. García (coord.), Procesos del Patrimonio Cultural (págs. 53-79). Guadalajara: El Colegio de Jalisco.

Gómez Consuegra, L. (2012). Reconfiguración del paisaje urbano histórico. . En AAVV, Paisaje cultural urbano e identidad territorial. $2^{\circ}$ coloquio red internacional de pensamiento crítico sobre globalización y patrimonio construido. (Vol. 2, págs. 1109-1120). Florencia, Italia: Aracne editrice.
Gómez, L., \& Alvarez, J. (2006). El carácter sistémico de la enseñanza de la conservación del patrimonio en la Universidad de Camagüey. En L. Gómez, \& G. Bezoari (coords), La Enseñanza de la Conservación del Patrimonio. Experiencias en la Universidad de Camagüey y en el Politécnico de Milán (págs. 1320, 31-42, 63-70). Milán, Italia: Politécnico de Milán.

Gómez, L., \& Gamboa, D. (1986). Tipología urbana del centro histórico de Camagüey. Arquitectura y Urbanismo, 7 (1), 40-45.

Gómez Consuegra, L., \& otros. (2013). Planeamiento del centro histórico de Camagüey. Estudios Jaliscienses (91), 47-59.

Gómez Consuegra, L. (2015). Conservación del centro histórico de Camagüey. En L. Gómez, \& O. Niglio (comp. y coord.), Conservación de centros históricos en Cuba (Vol. 1, págs. 269-295). Roma, Italia: Aracne editrice.

Gómez, L. (2018). El paisaje urbano histórico sostenible.¿Conservación o reconfiguración? En AAVV, Sostenibilidad ¿un extraño a la modernidad? Guadalajara, Jalisco, México: Universidad de Guadalajara.

Gómez Consuegra, L., \& Pascual Wong, T. (2007). La población como protagonista social en Camagüey: un análisis de los barrios El Carmen y San Juan de Dios. En A. Hearn (coord.), Cultura, Tradición, y Comunidad: Perspectivas sobre la Participación y el Desarrollo en Cuba (págs. 244-272). La Habana, Cuba: Imagen Contemporánea.

Gómez Consuegra, L., \& Pérez Justo, K. (2011). Reflexiones sobre patrimonio cultural. Lo inmaterial del centro histórico de Camagüey, Patrimonio Mundial. Apuntes, 24 (2), 260275.

Gomez, L., \& otros. (2009). Guía de Arquitectura y Paisaje de Camagüey y Ciego de Ávila. Sevilla, España: Junta de Andalucía.

Gómez Consuegra, L., \& otros. (2011). Contribución a la conservación del centro histórico de Camagüey. Premio ACC 2011. Multimedia. CD Rom. Camagüey, Cuba.

Gómez Consuegra, L., \& otros. (2015). El turismo en la zona Patrimonio Cultural de la Humanidad de Camagüey. IX Simposio Internacional Desafios en el Manejo y Gestión de Ciudades. Conferencia. Camagüey, Cuba.

Gómez Consuegra, L., \& otros. (2016). El medioambiente en la zona Patrimonio Cultural de la Humanidad de Camagüey. X Simposio Internacional Desafios en el Manejo y Gestión de Ciudades. Conferencia. Camagüey, Cuba.

Gómez Consuegra, L., \& otros. (2017). Impactos de la actividad por cuenta propia en el 
Paisaje urbano histórico de Camagüey. XI Simposio Internacional Desafios en el Manejo $y$ Gestión de Ciudades. Conferencia. Camagüey, Cuba.

Gómez Consuegra, L., \& otros. (2019). El paisaje urbano histórico y políticas públicas. XIII Simposio Internacional Desafios en el Manejo y Gestión de Ciudades. Conferencia. Camagüey, Cuba.

Gómez, L., \& Puente, A. (2015). Las Escuelas Pías, uno de los edificios más antiguos de Camagüey. . En E. Cento (coord.), Cuadernos de Historia Principeña No. 14 (págs. 89-107). Camagüey: Ácana.

Guerrero Muñoz, F. (2009). Modelo para la conservación del patrimonio cultural y natural; una alternativa con fines turísticos en el valle de Ameca, Jalisco, México. Tesis doctoral. Instituto Superior Politécnico "José A. Echeverría". ISPJAE, La Habana, Cuba.

Guzmán Laste, E. (2005). Proyecto Agenda 21 local para el centro histórico de la ciudad de Camagüey. Tesis de maestría. Universidad de Camagüey, Camagüey, Cuba.

Kaba, O. (2005). Rehabilitación integral de la vivienda del Barrio San Juan de Dios . Trabajo de diploma. Universidad de Camagüey, Camagüey, Cuba.

Lugones, M. (2008). Potencial de cambio de uso de un sector del centro histórico de Camagüey. Trabajo de diploma . Universidad de Camagüey, Camagüey, Cuba.

Machado, E. (1985). Delimitación del centro histórico de Camagüey. Trabajo de diploma . Universidad de Camagüey, Camagüey, Cuba.

Marichal, A. (2005). Modelo de estructuración para ciudades intermedias aplicando el patrón de crecimiento por sectores. Camagüey como caso de estudio. Tesis doctoral . Instituto Superior Politécnico "José A. Echeverría" ISPJAE, La Habana, Cuba.

Marín, A. (2005). Propuesta de regulaciones generales y específicas para los principales espacios públicos del centro histórico de Camagüey. Tesis de maestría. Universidad de Camagüey, Camagüey, Cuba.

Mas Sarabia, V. (2000). Caracterización del repertorio habitacional ecléctico del Centro Histórico de Camagüey. Tesis doctoral . Instituto Superior Politécnico "José A. Echeverría". ISPJAE, La Habana, Cuba.

Montero, C. (1990). Inventario general de monumentos. Verificación y estructuración. Trabajo de diploma. Universidad de Camagüey, Camagüey, Cuba.

Novoa, M. E. (2015). Valoración de las transformaciones de las viviendas en el centro histórico camagüeyano. Tesis de maestría . Universidad de Camagüey, Camagüey, Cuba.

Pascual, T., \& Gómez, L. (2014). Calle Maceo, un eje comercial tradicional y el proceso de reapropiación. En B. Nuñez Miranda, \& A. Peregrina (coords), Patrimonio y modos de vida (págs. 43-68). Zapopan, Jalisco, México: El Colegio de Jalisco.

Pérez Justo, K. (2010). Delimitación de los centros históricos. El Centro Histórico de Camagüey. Tesis de maestría. Universidad de Camagüey, Camagüey, Cuba.

Pintado, E. (2002). Prediagnóstico de la zona priorizada del centro histórico de Camagüey para un proyecto comunitario . Trabajo de diploma . Universidad de Camagüey, Camagüey, Cuba.

Plá Ponce de León, E. (2002). Centro histórico de Camagüey. Recurso para el turismo cultural. Tesis de maestría. Univerisdad de Camagüey, Camagüey, Cuba.

Prieto Herrera, O. (1991). Caracterización tipológica de la arquitectura domética camagüeyana. Tesis doctoral . Instituto Superior Politécnico "José A. Echeverría". ISPJAE, La Habana, Cuba.

Reyes, M. (1986). Recuperación del fondo habitable del casco histórico de Camagüey. Trabajo de diploma . Universidad de Camagüey, Camagüey, Cuba.

Rodríguez Algeciras, J. A. (2016). El paisaje urbano histórico del Área Patrimonio de la Humanidad de la ciudad de Camagüey: morfotipología y bioclima térmico. Tesis de maestría . Universidad de Camagüey, Camagüey, Cuba.

Rodriguez Algeciras, J. A., Gómez Consuegra, L., \& otros. (2016). Spatial-temporal study on the effects of urban street configurations on human thermal comfort in the world heritage city of Camagüey-Cuba. Building and Environment (The International Journal of Building Science and its Applications) (101), 85-101.

Rodríguez, K. (2005). Diagnóstico general y propuestas para la conservación del barrio de San Juan de Dios . Trabajo de Diploma . Universidad de Camagüey, Camagüey, Cuba.

Rossi, A. (1971). La arquitectura de la ciudad. Barcelona: Gustavo Gili.

Sariol, E. (1984). Sistema de indicadores y variables patrimoniales arquitectónicas y urbanas para el manejo de las potencialidades de uso en los inmuebles del centro histórico de Camagüey. Tesis de maestría. Universidad de Camagüey, Camagüey, Cuba.

Varona, M. (2005). Problemática medioambiental en los ejes urbanos principales de las zonas de protección 1 y 2 del centro histórico de la 
ciudad de Camagüey. Tesis de maestría . Universidad de Camagüey, Camagüey, Cuba.

WHC/UNESCO. (2008). Inscripción del centro Histórico de Camagüey (C1270), Cuba, en la lista del Patrimonio Mundial. WHC/74210.1/ns/mgl/176. Quebec, Canadá.

WHC/UNESCO. (2011). Recomendación sobre el paisaje urbano bistórico, con inclusión de un glosario de definiciones. Obtenido de http://whc.unesco.org/document/117660

Yanez, G., Gómez, L., \& otros. (2006). Manual Operativo de Conservación de Centros Históricos, Recursos Naturales y Medio Ambiente y Para el Empresario e Inversionista Turistico (3 tomos). Tlaxcala, México: El Colegio de Tlaxcala. A. C.

Ziccardi, A., \& otros. (2015). Procesos y actores de la planeación participativa en centros históricos de ciudades mexicanas. En AAVV, Conservación y desarrollo sustentable en centros históricos. México DF.: INAH, INBA, UNAM.

\section{Notas}

iEn Cuba, la Ley de Protección al Patrimonio Cultural, No. 1 de 1977, establece 4 grados de protección.

ii Sólo el 14,5\% de las manzanas son rectangulares o cuadradas.

iiiInstituto Nacional de Geodesia y Cartografía (GeoCuba), institución que se dedica a la informatización cartográfica del territorio cubano y sus instancias provinciales.

iv En los tres documentos participó la autora del presente trabajo

v Proyecto financiado por la Comunidad Autónoma de Islas Baleares.

vi Se conoce en Cuba, como cuarterías lo que en otros países latinoamericanos se denominan conventillos, inquilinato, vecindades, u otros. 